\title{
Identifying a possible new target for diagnosis and treatment of postmenopausal osteoporosis through bioinformatics and clinical sample analysis
}

\author{
Ting Liu ${ }^{1 \#}$, Jiajun Huang ${ }^{2 \#}$, Dongni $\mathrm{Xu}^{1}$, Yuxi $\mathrm{Li}^{2} \wedge$ \\ ${ }^{1}$ Department of Anesthesia, Sun Yat-sen Memorial Hospital, Sun Yat-sen University, Guangzhou, China; ${ }^{2}$ Department of Orthopedics, Sun Yat-sen \\ Memorial Hospital, Sun Yat-sen University, Guangzhou, China \\ Contributions: (I) Conception and design: Y Li; (II) Administrative support: D Xu; (III) Provision of study materials or patients: Y Li; (IV) Collection \\ and assembly of data: T Liu; (V) Data analysis and interpretation: J Huang; (VI) Manuscript writing: All authors; (VII) Final approval of manuscript: \\ All authors. \\ \#These authors contributed equally to this work. \\ Correspondence to: Dongni Xu. Department of Anesthesia, Sun Yat-sen Memorial Hospital, Sun Yat-sen University, Guangzhou 510120, China. Email: \\ xudongni@mail.sysu.edu.cn; Yuxi Li. Department of Orthopedics, Sun Yat-sen Memorial Hospital, Sun Yat-sen University, Guangzhou 510120, \\ China. Email: liyx73@mail.sysu.edu.cn.
}

Background: Postmenopausal osteoporosis, a common yet chronic systemic metabolic disease, has become a major public health problem due to life expectancy increasing around the world. The differentiation of mesenchymal stem cells (MSCs) into osteoblasts, and the differentiation of circulating monocyte cells into osteoclasts, play an important role in the balance of bone metabolism. However, when both undergo pathological changes, it can lead to abnormalities, resulting in osteoporosis. This study aims to explore a new biomarker for postmenopausal osteoporosis, thereby providing a new entry point for bioinformatic research into the clinical diagnosis and treatment of the disease.

Methods: Using the Gene Expression Omnibus (GEO) database, microarray analysis was conducted to identify differentially expressed genes in MSCs and monocytes in both postmenopausal osteoporosis patients and a healthy control group. The Database for Annotation, Visualization and Integrated Discovery (DAVID) database was used to analyze the function and enrichment of the selected genes, and a protein-protein interaction (PPI) network was constructed from the Search Tool for the Retrieval of Interacting Genes/ Proteins (STRING) website and displayed in Cytoscape. To achieve the final results, module analysis of the PPI network was performed by using Molecular Complex Detection (MCODE).

Results: We identified 45 high-expression and 26 low-expression genes through the study, all of which underwent pathway enrichment analysis. This enrichment was observed in the cell cycle regulation, osteoclast differentiation, tumor necrosis factor (TNF) signaling pathway, and RNA transport. The top 10 hub genes of the PPI network were SF3B1, SRSF5, FUBP1, SRSF3, TIA1, KHSRP, LUC7L3, PNN, SRC, and $A T R X$. Comparing the MSCs and monocytes between the postmenopausal osteoporosis patients and the healthy control group, we noted that the expression of the above genes differed greatly.

Conclusions: Through bioinformatic analysis and clinical specimen validation, our study provides a new way for exploring the pathogenesis of postmenopausal osteoporosis. Most importantly, it suggests that the hub genes, SF3B1, SRSF5, FUBP1, KHSRP, and SRC, may become new diagnostic markers and therapeutic targets for diagnosing and treating postmenopausal osteoporosis in the future.

^ ORCID: 0000-0002-4470-7679. 
Keywords: Postmenopausal osteoporosis; bioinformatics; gene expression

Submitted May 10, 2021. Accepted for publication Jul 14, 2021.

doi: 10.21037/atm-21-3098

View this article at: https://dx.doi.org/10.21037/atm-21-3098

\section{Introduction}

Postmenopausal osteoporosis is a chronic systemic metabolic disease associated with decreased estrogen levels. Symptoms are characterized by the thinning of a patient's bones, increasing the risk of fractures, particularly in the hip, wrist, and spine. As well as causing great pain to patients, osteoporotic fractures can also lead to disabilities, thereby putting more pressure on a country's public health system. In addition, associated complications, such as pressure sores, hypostatic pneumonia, and pulmonary embolism have been noted to result in nearly $30 \%$ of patients dying within a year of being diagnosed with the disease. As life expectancy increases around the world, it has been noted that the impact of this age-related disease will continue to expand. For example, in the United States it is estimated that by 2020, the population over the age of 50 will increase to 121.3 million, with over 3 million of those experiencing some sort of osteoporotic fracture. This means that the estimated cost of treating the disease in the United States alone will be nearly $\$ 25$ billion per year (1-5). In 1994, the World Health Organization (WHO) established diagnostic criteria for determining osteoporosis through a bone mineral density (BMD) test. Through this method, they ascertained that if a BMD T-score result was -2.5 or less and if the patient's low bone mass had a BMD T-score between -1 and -2.5 , then osteoporosis could be diagnosed. However, following more recent research on the disease, it has been noted that BMD tests are a somewhat limited clinical indicator for diagnosis, as determining osteoporosis requires an overall assessment of a patient's fracture risk. Several fracture risk assessment tools currently exist, including the Fracture Risk Assessment Tool (FRAX), Garvan Fracture risk calculator, and QFractureScores-2016. When used with a BMD test, these tools can help calculate a more accurate overall fracture risk (6).

Bone homeostasis is maintained and regulated by the complex interaction between osteoblasts and osteoclasts. Osteoblasts help to maintain and form healthy bones through protein synthesis and matrix secretion, while the main function of osteoclasts is bone resorption. In postmenopausal osteoporosis, there are 2 periods of bone loss: the first is a period of rapid loss, lasting 3 to 5 years (menopause-related bone loss), and the second is a period of slow loss, lasting 10 to 20 years (age-related bone loss). The link between menopause and osteoporosis stems from the fact estrogen suppresses apoptosis, thereby enhancing osteogenic differentiation of cultured mesenchymal stem cells (MSCs), promoting the osteoblast before and after osteoblast differentiation, thereby prolonging the life span of osteoblasts and bone cells. Estrogen can also interfere with the downstream signaling of osteoclasts, preventing osteoclast differentiation, which increases osteoblast apoptosis and transforming growth factor beta (TGF- $\beta$ ) estrogen deficiency-the main cause for the initial phase of rapid bone loss in postmenopausal women. In short, estrogen deficiency directly affects all bone cells by regulating cell differentiation activity and apoptosis, and indirectly affects all bone cells by changing the expression of estrogen response target genes, resulting in bone resorption increasing faster than bone metabolism. Research has also noted that osteoporosis, osteoporotic fractures, and low bone density are highly hereditary, with a heritability of 0.6 to 0.8 , meaning that $60-80 \%$ of bone density changes in these individuals are inherited from their parents, while the heritability of osteoporotic fractures is 0.5 to 0.7 (7). Therefore, identifying the individual variations and genes that contribute to osteoporosis and determining how they interact to influence molecular networks and system functions may provide new targets and markers for the diagnosis, drug development, and treatment evaluation of osteoporosis in postmenopausal women.

As a high-throughput and efficient sequencing technology, gene microarray analysis is widely used to screen important genetic or epigenetic changes in the development of various diseases and to identify biomarkers related to the diagnosis or evaluation of patient prognosis (8). For this study however, we have used both bioinformatic tools, as well as microarray analysis, to reanalyze gene expressions. This approach allowed us to better identify the relation between MSCs in the bones of postmenopausal osteoporosis patients and the mononuclear macrophages of differentially expressed genes (DEGs). This 
process included using biological process (BP) annotation and biological pathway enrichment analysis to construct a protein-protein interaction (PPI) network of DEGs, which then allowed for the module analysis of hub genes in the PPI network. After the identification of these hub gene expressions in the MSCs and mononuclear macrophages of both postmenopausal osteoporosis patients and a healthy control group, the results were then compared. Overall, the aim of this study was to determine the abnormal expression of genes and pathways in both the MSCs and mononuclear macrophage of patients, through which new ways to study the pathogenesis of postmenopausal osteoporosis may be discovered. We present the following article in accordance with the MDAR reporting checklist (available at https:// dx.doi.org/10.21037/atm-21-3098).

\section{Methods}

\section{Microarray data}

Three gene expressing profiling data sets (GSE56815, GSE2208, and GSE35958) were obtained from the Gene Expression Omnibus (GEO), a public repository for data storage (www.ncbi.nlm.nih.gov/geo). The dataset GSE56815, based on GPL96 (HG-U133A) Affymetrix Human Genome U133A Array, included peripheral blood monocytes (PBMs) from 40 postmenopausal women, 20 of whom had osteoporosis. The next data set, GSE2208, based on GPL96 (HG-U133A) Affymetrix Human Genome U133A Array, included PBMs from 19 women, 9 of whom had osteoporosis. The final data set, GSE35958, based on GPL570 (HG-U133_Plus_2) Affymetrix Human Genome U133 Plus 2.0 Array, included MSCs isolated and cultured from bone marrow in the hips of 8 postmenopausal women, 5 of whom had osteoporosis.

\section{Data processing}

To analyze the microarray data, GEO's interactive web tool, GEO2R, was used to select and compare samples from differentially expressed denes (DEG). Using the following filter criteria, $\mathrm{P}<0.05$ and $|\mathrm{t}|>2$, we were able to identify DEGs and distinguish them through up- and downregulated expressions. We then used the MATCH function to identify overlapping up- and downregulated DEGs in the 3 previously mentioned data sets (GSE56815, GSE56814, and GSE35958).

\section{Functional and pathway enrichment analysis}

Using the Database for Annotation, Visualization, and Integrated Discovery (DAVID), the up- and downregulated expression genes underwent Gene Ontology (GO) analysis. This involved examining each gene's cellular components, molecular function (MF), and BPs. Pathway enrichment analysis was then conducted using the Kyoto Encyclopedia of Genes and Genomes (KEGG) $(9,10)$. A P value $<0.05$ was considered statistically significant.

\section{PPI network construction and module analysis}

To further examine the biological function of the upand downregulated DEGs in patients suffering from postmenopausal osteoporosis, a PPI network was created using the Search Tool for Retrieval of Interaction Genes (STRING) database (an interaction score of 0.4 was regarded as the cutoff criterion). Subsequently, the Molecular Complex Detection (MCODE) in Cytoscape software was used to screen modules in the PPI network, with an MCODE score $>3$ and a number of nodes $>4$ being required. Hub genes were screened by a connection degree $>10$. The functional enrichment analysis of each module gene was performed by using DAVID, and the significance threshold was a $\mathrm{P}$ value $<0.05$.

\section{Verification of the bub genes in MSCs and monocytes from postmenopausal women with osteoporosis and normal control group}

The study was in accordance with the Declaration of Helsinki (as revised in 2013). This study was approved by the Ethics Committee of Sun Yat-sen Memorial Hospital, Sun Yat-sen University. All participants signed the relevant consent forms. Postmenopausal women over the age of 60 who were admitted the hospital's Department of Orthopedics between October 2018 to January 2020 were included in the study. Patients were excluded from the study if they were found to suffer from any of the following: severe cardiopulmonary disease; untreated clotting disorders; systemic or spinal infections, history of malignant tumor, untreated bone metabolic diseases other than osteoporosis, digestive dysfunction, severe liver and/or kidney dysfunction, history of spinal or hip surgery, 25 -hydroxy vitamin $\mathrm{D}<23 \mathrm{nmol} / \mathrm{L}$, low quantity of serum calcium, abnormal levels of parathyroid 
hormone or thyroxine, history of fluoride use at therapeutic doses, history of estrogen replacement therapy or selective estrogen receptor agonist treatment, history of glucocorticoid use, and a history of antidepressant use. All patients who showed a decrease in BMD of more than 2.5 SD were included in the experimental group, while the other patients were included in the control group. The BMD level was measured using dual-energy $\mathrm{X}$-ray absorptiometry. Ultimately, 6 postmenopausal women with osteoporosis and 6 healthy postmenopausal females were included in the study.

\section{Sample collection and processing}

Through density gradient centrifugation, MSCs were first isolated from the bone marrow aspirates of a patients' spinous process or posterior superior iliac spine during spinal or spinal-pelvic fusion. After identifying MSC immunophenotype markers by flow cytometry, passages 3 to 5 were used for the study. Each patient also had $60 \mathrm{~mL}$ of peripheral blood collected by a qualified phlebotomist. During this process, ethylenediaminetetraacetic acid (EDTA) was used as an anticoagulant, and PBMs were immediately separated from the fresh blood samples. Density gradient centrifugation with a Histopaque-1077 (H1077-1, Sigma-Aldrich, St. Louis, MO, USA) was conducted to isolate the peripheral blood mononuclear cells (PBMCs) from the whole blood. The PBMs were then isolated from the PBMCs using a monocyte negative isolation kit (Dynal Biotech Inc., Lake Success, NY, USA).

\section{Total RNA extraction and real-time quantitative polymerase chain reaction}

Using TRIzol Reagent (Life Technologies Inc., USA), RNA was extracted from the blood samples to explore the expression of DEGs in both MSCs and PBMCs. To carry out the synthesis of cDNA (RT-PCR), we used the SuperScriptH III First-Strand Synthesis System (Invitrogen, USA). The quantitative real-time polymerase chain reaction (qRT-PCR) analysis was completed through a Light Cycler 480 Real-Time PCR System (Roche, Basel, Switzerland) using a SYBR Premix Ex Taq II kit (Takara, Otsu, Japan). The protocol for quantitative PCR assay was $95^{\circ} \mathrm{C}$ for 30 seconds, followed by 40 cycles at $95{ }^{\circ} \mathrm{C}$ for 5 seconds and $60{ }^{\circ} \mathrm{C}$ for 20 seconds. This method was repeated 3 times to calculate the mean messenger (mRNA) levels, while a fusion curve analysis was performed to determine the specific amplification of the target. The relative amount or fold change of the target genes was normalized according to the expression of the housekeeping gene, $\beta$-actin.

\section{Statistical analysis methods}

Statistical analysis was performed using SPSS20.0 statistical software (SPSS Inc., Chicago, IL, USA). The data was expressed as mean \pm standard deviation $\left(\bar{x}_{ \pm s}\right)$. The comparison between two groups was performed using $t$ test and the comparison among multiple groups was performed with one-way ANOVA analysis. $\mathrm{P}<0.05$ was considered statistically significant.

\section{Results}

\section{Identification of DEGs in postmenopausal osteoporosis}

Using the online software tool, GEO2R, to carry out microarray analysis, we identified 69 overlapping upregulated genes (2,409 in GSE56815, 492 in GSE2208) and 30 overlapping downregulated genes $(1,404$ in GSE56815, 296 in GSE2208) in peripheral monocytes. In the GSE35958 data set, 2,324 upregulated genes and 5,408 downregulated genes were identified in MSCs. Following this, we identified 45 significantly upregulated genes in both the MSCs and peripheral monocyte cells by overlapping 147 upregulated genes in the peripheral monocytes, and 2,324 upregulated genes in MSCs. Using the same method, we also discovered 26 significantly downregulated genes in both the MSCs and peripheral monocytes. These results can be seen in Figure 1. A representative heat map of GSE56815 (top 50 up- and downregulated genes) is shown in Figure 2.

\section{GO functional enrichment analysis}

The top 5 results from our GO enrichment analysis (displayed through DAVID) are shown in Table 1. These abnormally expressed genes in both MSCs and PBMs were enriched in BPs of regulation of mRNA splicing (via spliceosome), regulation of cell cycle, RNA splicing, negative regulation of viral genome replication, and mRNA splicing (via spliceosome). Regarding the cell components (CC), genes showed enrichment in the nucleoplasm, nuclear speck, cytoplasm, cytosol, and the nucleus. MF was also enriched in protein binding, poly(A) RNA binding, RNA binding, protein kinase activity, and ATP binding. 

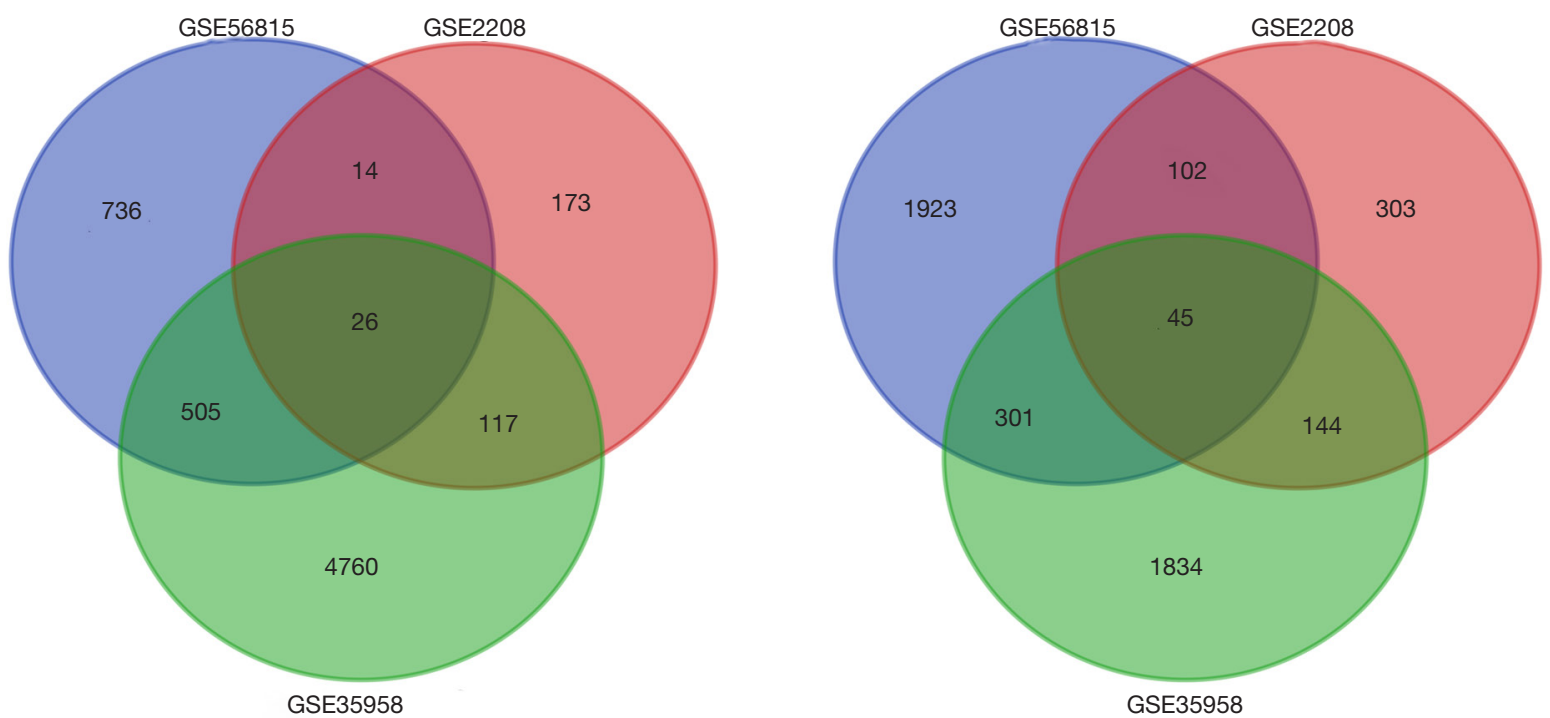

Figure 1 Identification of DEGs in gene expression data sets (GSE56815, GSE2208, and GSE35958). (A) Upregulated genes in both MSCs and peripheral blood monocytes; (B) downregulated genes in both MSCs and peripheral blood monocytes. MSC, mesenchymal stem cell; DEG, differentially expressed gene.

\section{KEGG patbway analysis}

Through using KEGG pathway enrichment analysis (Table 2), we were able to determine that the abnormally expressed genes in both MSCs and PBMs were significantly enriched in pathways of RNA transport, Herpes simplex infection, osteoclast differentiation, influenza A, and tuberculosis.

\section{PPI network construction, module analysis, and bub gene selection}

PPI networks were created using the STRING database and subsequently underwent module analysis through Cytoscape's MCODE software. Figure 3 illustrates how these PPI networks and modules were used to identify abnormally expressed genes in both the MSCs and PBMs of patients suffering from osteoporosis. These significant core modules also demonstrate the functions of mRNA splicing (via spliceosome) mRNA 3 '-end processing, and spliceosomal complex assembly. The top 5 hub genes were SF3B1, SRSF5, FUBP1, KHSRP, and SRC.

\section{Validation of the hub genes in MSCs and monocytes from postmenopausal women with osteoporosis and the control group}

To represent our analysis of postmenopausal osteoporosis, we compared the results of the qRT-PCR with bioinformatic analysis. In Figure 4, the expressions of the aforementioned hub genes (SF3B1, SRSF5, FUBP1, $K H S R P$, and $S R C$ ) were all consistent with our integrated analysis.

\section{Discussion}

Postmenopausal osteoporosis is a chronic systemic metabolic disease associated with decreased estrogen levels. The symptoms of the disease are characterized by an imbalance in bone metabolism as the result of several genetic, epigenetic, and environmental factors. According to the World Health Organization definition of osteoporosis based on bone density: osteoporosis is defined as a BMD 2.5 SD or more below the average value for premenopausal women (4). Normal BMD is defined as T-score of 1.0 or higher and a T-score between 1.0 and 2.5 is defined as osteopenia or low bone mass. Fifty percent of women $>50$ years of age will experience an osteoporotic bone fracture during their life time (4). Vitamin D3 and calcium are important aspects of osteoporosis prevention. Meanwhile, vitamin D has been found to play a key role in both direct (tumor gene expression, proliferation, and susceptibility to aggressive chemotherapy, etc.) and indirect (influence on tumor microenvironment and immune regulation) tumor suppressive mechanisms in the prostate, colon, breast, lung and skin cultures, cell lines expressing 


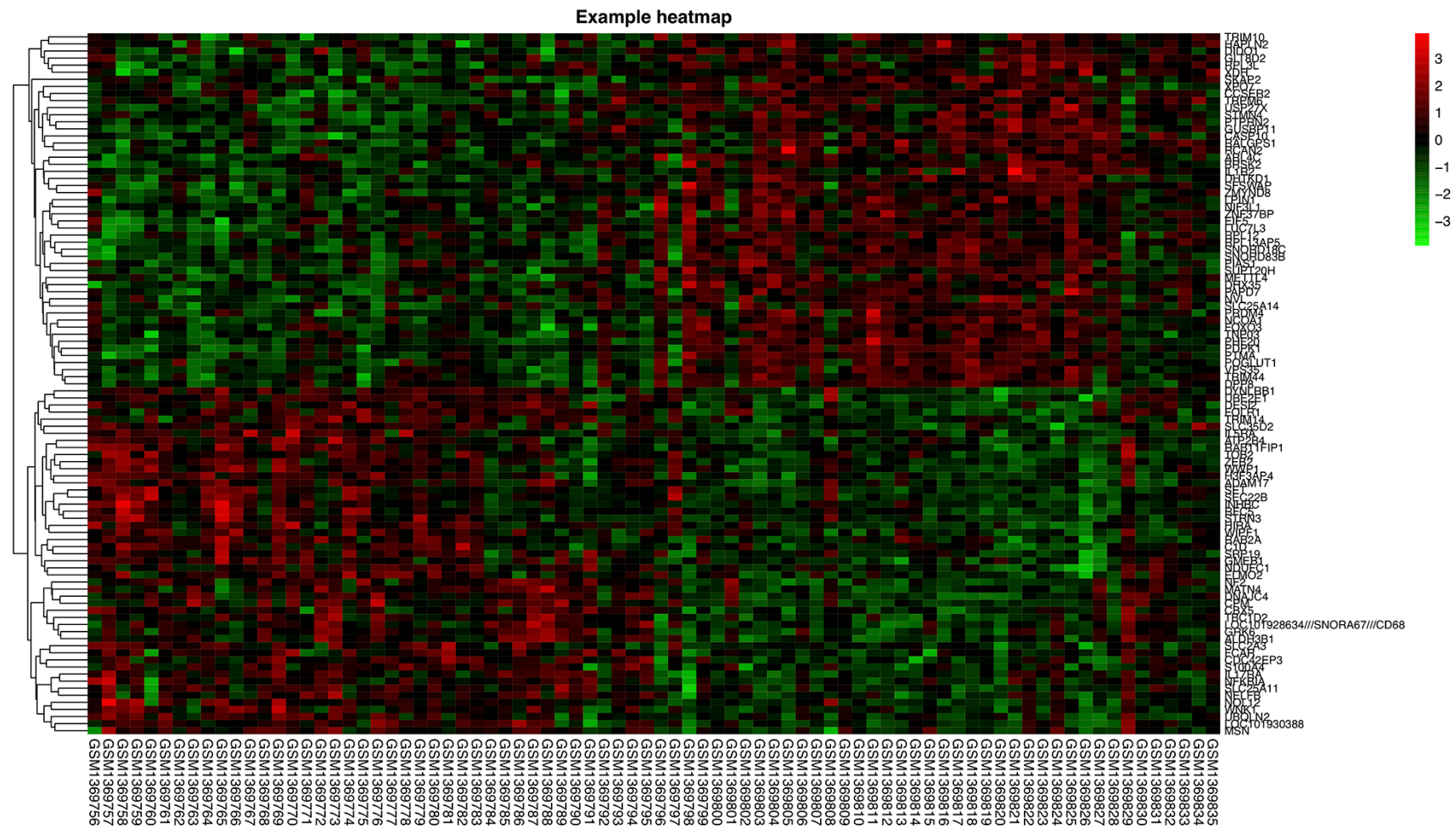

Figure 2 Representative heat map of the top 100 differentially expressed genes in the GSE56815 data set, including 50 upregulated and 50 downregulated genes. Note: red signifies upregulated genes, while green represents downregulated genes.

Table 1 Gene Ontology analysis of abnormally expressed genes in both mesenchymal stem cells and peripheral blood monocytes

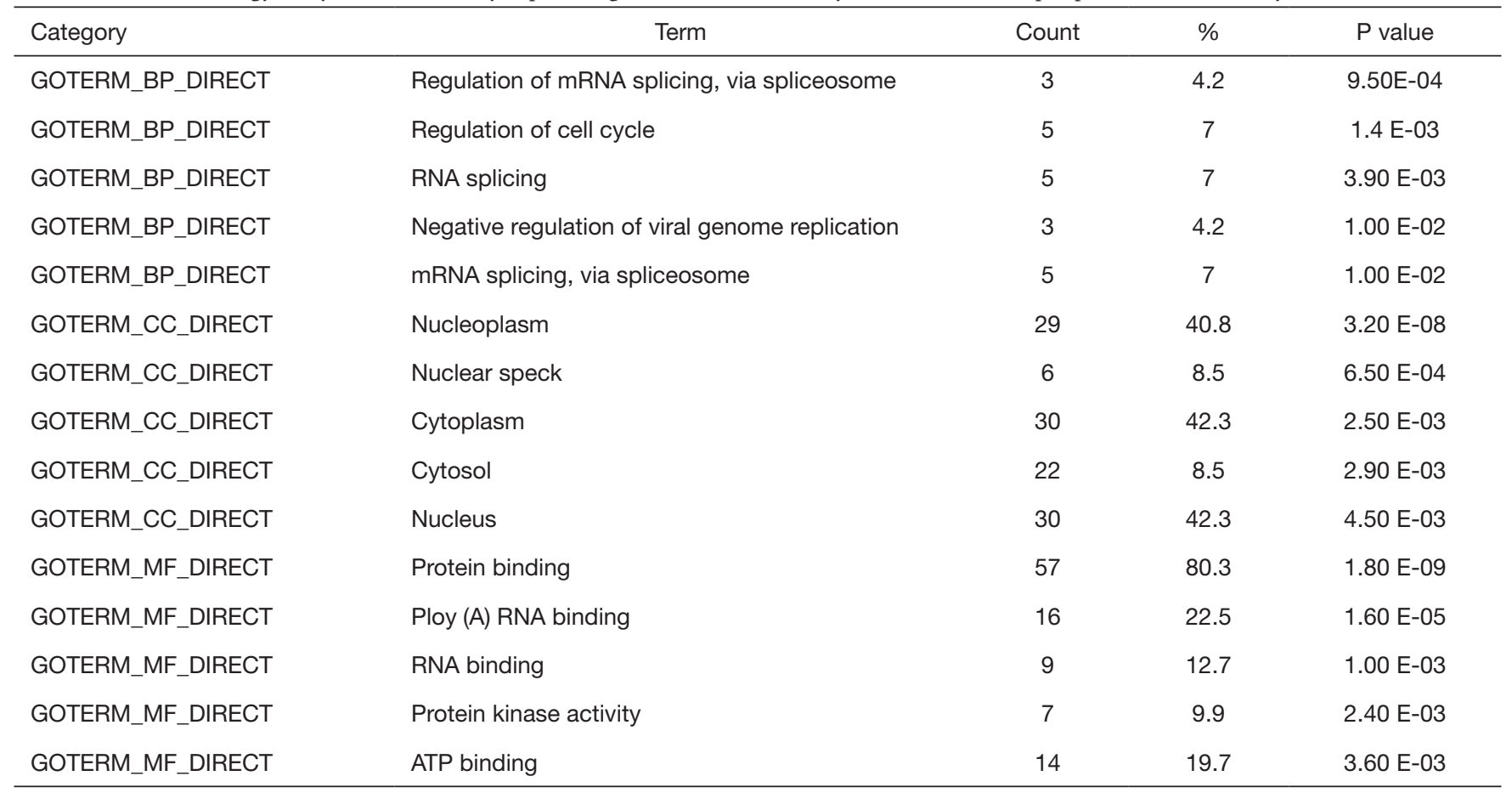


Table 2 Kyoto Encyclopedia of Genes and Genomes (KEGG) pathway analysis of abnormally expressed genes in both mesenchymal stem cells and peripheral blood monocytes

\begin{tabular}{lccccc}
\hline Pathway ID & Pathway name & Gene No. & $\%$ & P value & Genes \\
\hline hsa03013 & RNA transport & 5 & 7 & $7.00 \mathrm{E}-03$ & RANGAP1, EIF5, EIF5B, NUP50, PNN \\
hsa05168 & Herpes simplex infection & 5 & 7 & $8.70 \mathrm{E}-03$ & IFNAR1, SRSF3, SRSF5, SOCS3, TNF \\
hsa04380 & Osteoclast differentiation & 4 & 5.6 & $2.10 \mathrm{E}-02$ & JUNB, IFNAR1, SOCS3, TNF \\
hsa05164 & Influenza A & 4 & 5.6 & $4.30 \mathrm{E}-02$ & MALT1, SRC, CTSD, TNF \\
hsa05152 & Tuberculosis & 4 & 5.6 & $4.40 \mathrm{E}-02$ & MALT1, SRC, CTSD, TNF \\
\hline
\end{tabular}

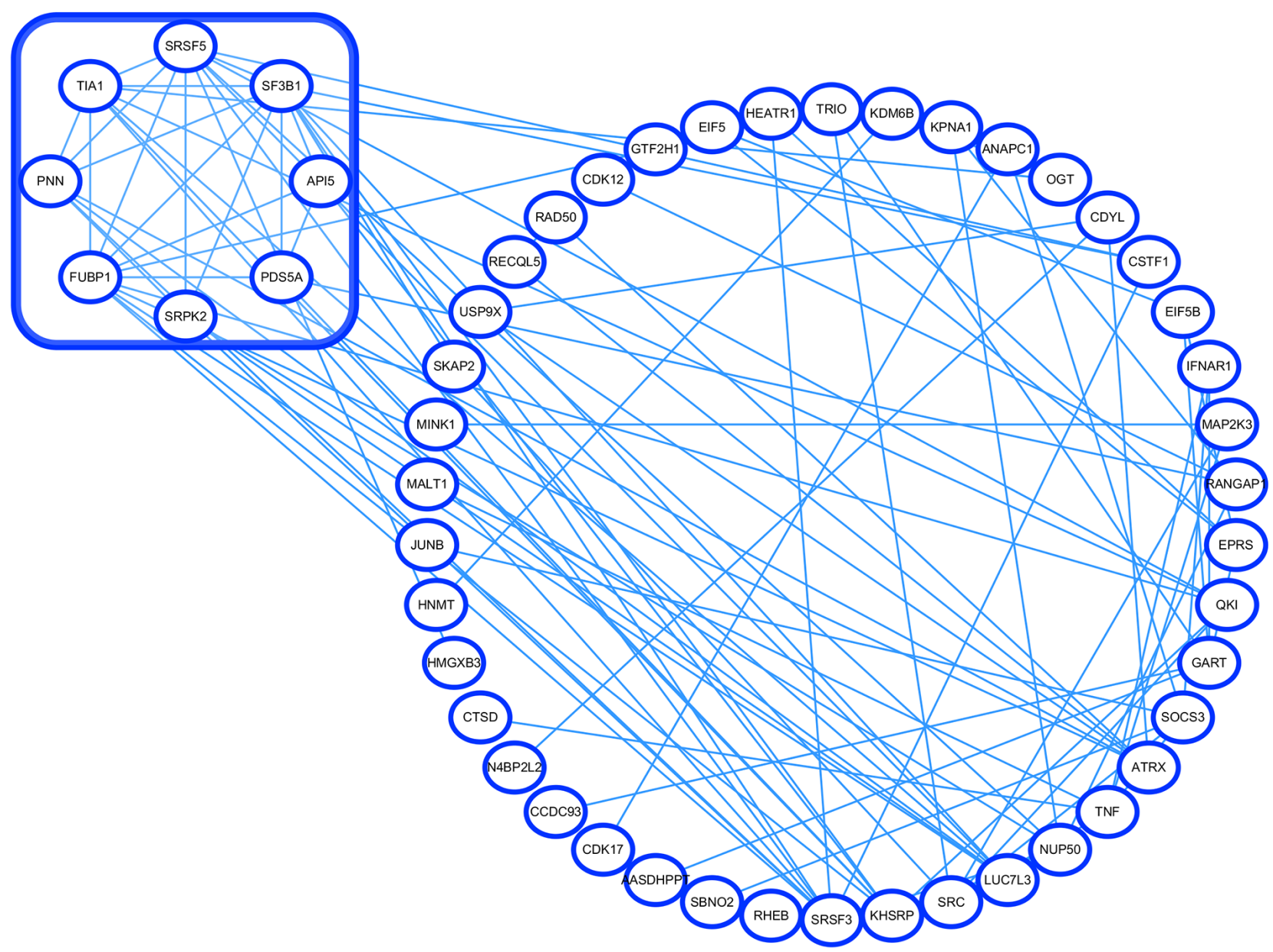

Figure 3 Protein-protein interaction (PPI) network and module of abnormally expressed genes in both mesenchymal stem cells and peripheral blood monocytes. 

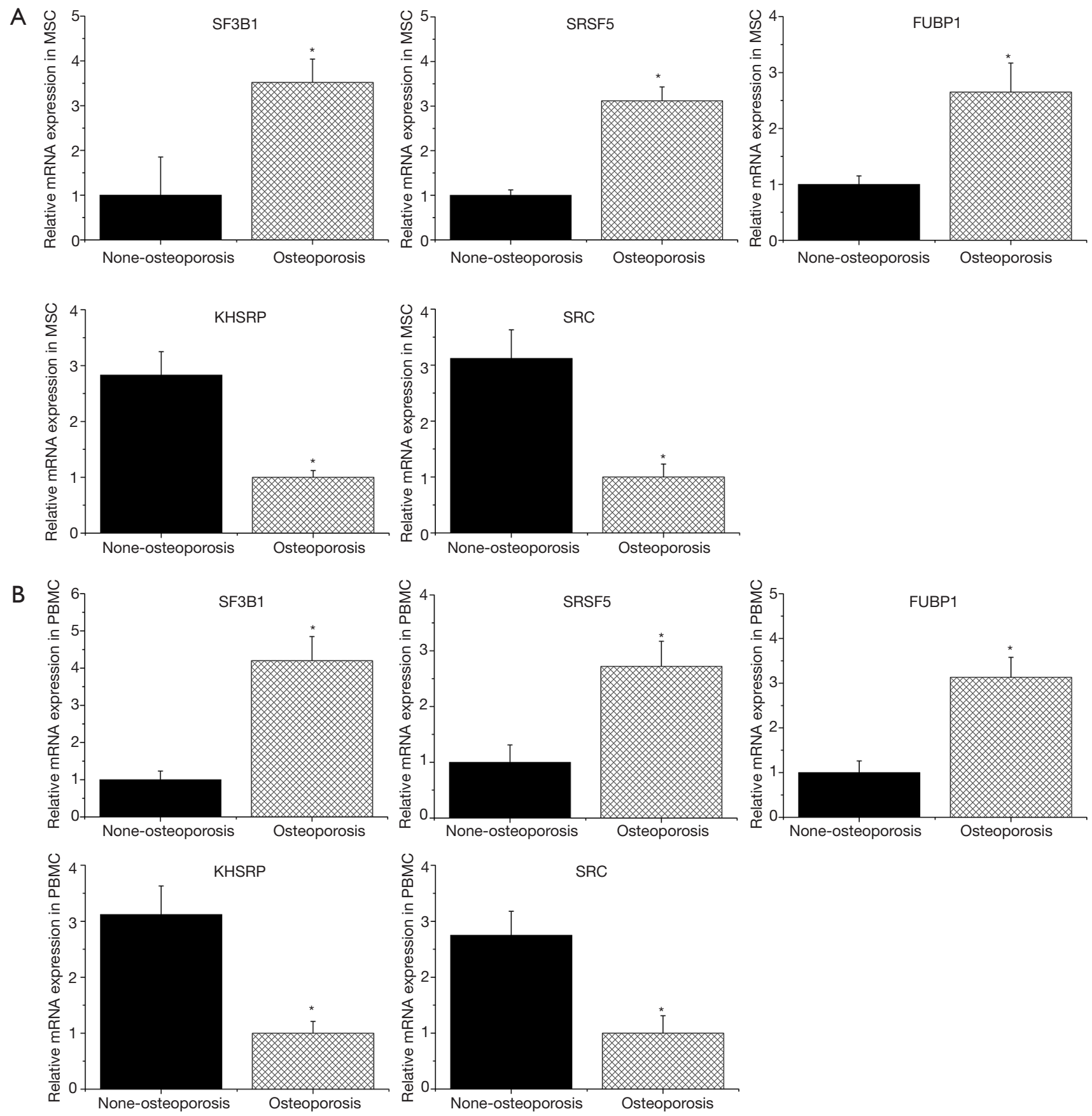

Figure 4 Quantitative real-time polymerase chain reaction verification results for postmenopausal osteoporosis. Section A and Section B represent the expression of hub genes in the both mesenchymal stem cells and peripheral blood mononuclear cells (PBMCs) of the 2 study groups, respectively. *, $\mathrm{P}<0.05$.

vitamin D receptors exposed to 1, 25-dihydroxyvitamin $\mathrm{D}$ and cell proliferation was inhibited (11). However, vitamin $\mathrm{D}$ and calcium intake have not been shown to reduce the risk of ovarian cancer, according to a recent research (12). Currently, the treatment of osteoporosis has been conducted with Bisphosphonates, Estrogen/ Selective Estrogen Receptor, Modulators, Denosumab and teriparatide to reduce the incidence of osteoporotic fracture 
(13-17). Bisphosphonates, an antiresorptive drug such as alendronate, reduced vertebral fractures by $47 \%$ and hip fractures by $51 \%$, while zoledronate maintains a patient's bone mass at a very low dose. And inhibits the osteoclastactivating factor receptor activator of nuclear factor kappa-B Ligand (RANKL) also effectively reduced the incidence of fracture in patients with osteoporosis, but there was obvious rebound in osteoclast mediated bone resorption after the discontinuation of Denosumab. Now Patient who have discontinued or will discontinue was advised to switch to other antiresorptive therapy within six months, but the curative effect is unclear. Drug therapy with oral or topical estrogens has anti-absorption and anabolic effects in the skeleton. However, due to other non-skeletal problems, the use of estrogen for the prevention and treatment of osteoporosis has declined sharply. Bone anabolic agents such as teriparatide, which is effective in increasing bone mass ( $9 \%$ increase in lumbar bone density; Femoral neck bone density increased by $3 \%$ ) and reduced the incidence of fractures (vertebral fractures decreased by $63 \%$; Nonvertebral fractures decreased by $53 \%$ ). These drugs offer a variety of treatment options for anti-osteoporosis treatment. In recent years, research on the cellular and molecular mechanisms of postmenopausal osteoporosis have revealed that a large number of genes and proteins are related to the occurrence and development of the disease. However, the effect such molecular mechanisms have on the development of osteoporosis remains unclear (6). Therefore, there exists a need in the research field to further explore the genes and/or proteins related to postmenopausal osteoporosis and detect the molecular mechanism of their interactions. In this study, we identified 45 upregulated genes and 26 downregulated genes in both MSCs and PBMs by using bioinformatic tools to analyze specific gene expression microarrays (GSE56815, GSE2208, and GSE35958) in patients postmenopausal osteoporosis. The subsequent result of our DAVID analysis revealed that these abnormally expressed genes were significantly enriched in processes of mRNA splicing regulation and cell cycle regulation. KEGG pathway enrichment analysis also indicated a significant enrichment in pathways including RNA transport and osteoclast differentiation, leading us to surmise that estrogen deficiency may induce abnormal microRNA (miRNA) transcriptions, which in turn, affect osteoclast differentiation and cell cycles.

After creating our PPI network, we determined that the top 5 hub genes were SF3B1, SRSF5, FUBP1, KHSRP, and $S R C$. By comparing the expression of these genes in MSCs and PMSCs of postmenopausal osteoporosis patients and a healthy group of postmenopausal women, the results were consistent with our analysis. The spliceosome, a large and dynamic multimegadalton small nuclear ribonucleoprotein composed of small nuclear RNAs associated with proteins, is responsible for removing introns from precursor mRNA (pre-mRNA) and generating mature, spliced mRNAs. To further elucidate the significance of this research, it is important to clarify the role of each of these 5 genes. For example, SF3B1, is a key subunit of the U2 small nuclear ribonucleoprotein ( $\mathrm{snRNP}$ ) which is required for the correct assembly of the splicing complex to the branch-point sequence $(18,19)$. At present, research on this gene has remained focused on its relation to tumors and the pathogenesis of myelodysplastic syndrome. This research has determined that recurrent somatic mutations in SF3B1 have been detected in human cancers, including hematological malignancies and solid tumors, and is thus pertinent to prognosis. Another hub gene, SRSF5, is a member of the serine/arginine (SR)-rich family of premRNA splicing factors, which constitute part of the spliceosome. It has been identified as a glucose-inducible protein that promotes tumor cell growth (20). The hub gene, FUBP 1 , is a master regulator of transcription, splicing, and translating via its bindings to single-stranded DNA (ssDNA) and RNA. It is an important activator or inhibitor of the transcription and translation of its target genes and promotes cell proliferation, inhibits cell apoptosis, and enhances cell migration by regulating complex networks (21). The overexpression of FUBP1 can lead to changes in the expression of its target genes, such as abnormal expressions of oncogene MYC. This MYC-ERR $\alpha$ pathway drives metabolic reprogramming during osteoclast differentiation and is an important regulator of physiological and pathological bone loss (22). Different spatial-temporal expression patterns of FUBP1 found in bone may be closely related to postmenopausal osteoporosis. KHSRP (KHtype splicing regulatory protein), also known as KSRP, is a single-stranded nucleic acid-binding protein (23). It has been observed that its interaction with SMAH in multipotent mesenchymal $\mathrm{C} 2 \mathrm{C} 12$ cells impairs their ability to bind to primary myogenic miRNAs (commonly called myomiRs) and promote their maturation. This hub gene also prevents myomiR maturation from promoting osteoblastic differentiation of $\mathrm{C} 2 \mathrm{C} 12$ cells. Atherosclerosis and osteoporosis is thought to have a common risk factors and mechanism of inflammation, suggest they may have comorbidities. KHSRP has proven to be of low density lipoprotein receptor unstable RNA binding protein, 


\section{Page 10 of 11}

inhibits the formation of low density lipoprotein receptor, which affects lipid metabolism, leading to atherosclerosis of the arteries (24-26). Thus, KHSRP may be a biomarker to identify the association between the two diseases. The last of the top 5 hub genes identified was $S R C$, a nonreceptor protein tyrosine kinase that is widely expressed in vertebrate cells. The presence of this protein in the brain, osteoclasts, and platelets has been noted to be at a level 5,200 times higher than in most other cells. Studies have shown that when $\mathrm{E} 2$ binds to $\mathrm{ER} \alpha$, SRC forms the complex, SHP2-c-Src, in a non-genomic manner which weakens RANKL stimulation-induced c-Src activation, resulting in impaired actin ring formation and reduced bone reabsorption (27). At the same time, Src was also considered as one of the important protein targets of Rhizoma Drynariae in a network pharmacological study of Rhizoma Drynariae, a traditional Chinese medicine with anti-inflammatory and anti-oxidative effects on bone (28-31). To conclude, through combining bioinformatic analysis with gene expression and microarray analysis, our study has discovered the abnormal expression of genes and pathways in patients of postmenopausal osteoporosis. This discovery puts researchers one step closer to determining how the molecular mechanism of osteoporosis develops in postmenopausal women.

\section{Acknowledgments}

Funding: This work was supported by the Sun Yat-sen Memorial Hospital Yat-sen Qihang Youth Project (No. YXQH202009).

\section{Footnote}

Reporting Checklist: The authors have completed the MDAR reporting checklist. Available at https://dx.doi. org/10.21037/atm-21-3098

Data Sharing Statement: Available at https://dx.doi. org/10.21037/atm-21-3098

Conflicts of Interest: All authors have completed the ICMJE uniform disclosure form (available at https://dx.doi. org/10.21037/atm-21-3098). All authors reported that this work was supported by the Sun Yat-sen Memorial Hospital Yat-sen Qihang Youth Project (No. YXQH202009). The authors have no other conflicts of interest to declare.
Ethical Statement: The authors are accountable for all aspects of the work in ensuring that questions related to the accuracy or integrity of any part of the work are appropriately investigated and resolved. The study was in accordance with the Declaration of Helsinki (as revised in 2013). This study was approved by the Ethics Committee of Sun Yat-sen Memorial Hospital, Sun Yat-sen University. All participants signed the relevant consent forms.

Open Access Statement: This is an Open Access article distributed in accordance with the Creative Commons Attribution-NonCommercial-NoDerivs 4.0 International License (CC BY-NC-ND 4.0), which permits the noncommercial replication and distribution of the article with the strict proviso that no changes or edits are made and the original work is properly cited (including links to both the formal publication through the relevant DOI and the license). See: https://creativecommons.org/licenses/by-nc-nd/4.0/.

\section{References}

1. Black DM, Rosen CJ. Clinical Practice. Postmenopausal Osteoporosis. N Engl J Med 2016;374:254-62.

2. Burge R, Dawson-Hughes B, Solomon DH, et al. Incidence and economic burden of osteoporosis-related fractures in the United States, 2005-2025. J Bone Miner Res 2007;22:465-75.

3. Rachner TD, Khosla S, Hofbauer LC. Osteoporosis: now and the future. Lancet 2011;377:1276-87.

4. Lewiecki EM. Romosozumab, clinical trials, and realworld care of patients with osteoporosis. Ann Transl Med 2020;8:974.

5. US Preventive Services Task Force; Curry SJ, Krist AH, et al. Screening for Osteoporosis to Prevent Fractures: US Preventive Services Task Force Recommendation Statement. JAMA 2018;319:2521-31.

6. Compston JE, McClung MR, Leslie WD. Osteoporosis. Lancet 2019;393:364-76.

7. Yang TL, Shen H, Liu A, et al. A road map for understanding molecular and genetic determinants of osteoporosis. Nat Rev Endocrinol 2020;16:91-103.

8. Al-Barghouthi BM, Farber CR. Dissecting the Genetics of Osteoporosis using Systems Approaches. Trends Genet 2019;35:55-67.

9. Dennis G Jr, Sherman BT, Hosack DA, et al. DAVID: Database for Annotation, Visualization, and Integrated Discovery. Genome Biol 2003;4:P3. 
10. Yang C, Ren J, Li B, et al. Identification of gene biomarkers in patients with postmenopausal osteoporosis. Mol Med Rep 2019;19:1065-73.

11. Bilani N, Elson L, Szuchan C, et al. Newly-identified Pathways Relating Vitamin D to Carcinogenesis: A Review. In Vivo 2021;35:1345-54.

12. $\mathrm{Xu} \mathrm{J}$, Chen $\mathrm{K}, \mathrm{Zhao} \mathrm{F}$, et al. Association between vitamin $\mathrm{D} /$ calcium intake and 25-hydroxyvitamin D and risk of ovarian cancer: a dose-response relationship meta-analysis. Eur J Clin Nutr 2021;75:417-29.

13. Słupski W, Jawień P, Nowak B. Botanicals in Postmenopausal Osteoporosis. Nutrients 2021;13:1609.

14. Gosset A, Pouillès JM, Trémollieres F. Menopausal hormone therapy for the management of osteoporosis. Best Pract Res Clin Endocrinol Metab 2021. [Epub ahead of print]. doi: 10.1016/j.beem.2021.101551.

15. Arceo-Mendoza RM, Camacho PM. Postmenopausal Osteoporosis: Latest Guidelines. Endocrinol Metab Clin North Am 2021;50:167-78.

16. Noble JA, McKenna MJ, Crowley RK. Should denosumab treatment for osteoporosis be continued indefinitely? Ther Adv Endocrinol Metab 2021;12:20420188211010052.

17. Saul D, Drake MT. Update on Approved Osteoporosis Therapies Including Combination and Sequential Use of Agents. Endocrinol Metab Clin North Am 2021;50:179-91.

18. Obeng EA, Chappell RJ, Seiler M, et al. Physiologic Expression of Sf3b1(K700E) Causes Impaired Erythropoiesis, Aberrant Splicing, and Sensitivity to Therapeutic Spliceosome Modulation. Cancer Cell 2016;30:404-17.

19. Zhou Z, Gong Q, Wang Y, et al. The biological function and clinical significance of SF3B1 mutations in cancer. Biomark Res 2020;8:38.

20. Chen Y, Huang Q, Liu W, et al. Mutually exclusive acetylation and ubiquitylation of the splicing factor SRSF5 control tumor growth. Nat Commun 2018;9:2464.

21. Debaize L, Troadec MB. The master regulator FUBP1: its emerging role in normal cell function and malignant development. Cell Mol Life Sci 2019;76:259-81.

22. Bae S, Lee MJ, Mun SH, et al. MYC-dependent oxidative metabolism regulates osteoclastogenesis via nuclear receptor ERR $\alpha$. J Clin Invest 2017;127:2555-68.

23. Briata P, Bordo D, Puppo M, et al. Diverse roles of the nucleic acid-binding protein KHSRP in cell differentiation and disease. Wiley Interdiscip Rev RNA 2016;7:227-40.

24. Carmona-Fernandes D, Barreira SC, Leonardo N, et al. Atherosclerosis and Bone Loss in Humans-Results From Deceased Donors and From Patients Submitted to Carotid Endarterectomy. Front Med (Lausanne) 2021;8:672496.

25. Jiang H, Zhang J, Du Y, et al. microRNA-185 modulates low density lipoprotein receptor expression as a key posttranscriptional regulator. Atherosclerosis 2015;243:523-32.

26. Mishra BH, Mishra PP, Mononen N, et al. Uncovering the shared lipidomic markers of subclinical osteoporosisatherosclerosis comorbidity: The Young Finns Study. Bone 2021;151:116030.

27. Park HJ, Gholam-Zadeh M, Yoon SY, et al. Estrogen Decreases Cytoskeletal Organization by Forming an ER $\alpha /$ SHP2/c-Src Complex in Osteoclasts to Protect against Ovariectomy-Induced Bone Loss in Mice. Antioxidants (Basel) 2021;10:619.

28. Gan D, Xu X, Chen D, et al. Network PharmacologyBased Pharmacological Mechanism of the Chinese Medicine Rhizoma drynariae Against Osteoporosis. Med Sci Monit 2019;25:5700-16.

29. Mu P, Hu Y, Ma X, et al. Total flavonoids of Rhizoma Drynariae combined with calcium attenuate osteoporosis by reducing reactive oxygen species generation. Exp Ther Med 2021;21:618.

30. Shen Z, Chen Z, Li Z, et al. Total Flavonoids of Rhizoma Drynariae Enhances Angiogenic-Osteogenic Coupling During Distraction Osteogenesis by Promoting Type $\mathrm{H}$ Vessel Formation Through PDGF-BB/PDGFR- $\beta$ Instead of HIF-1 $\alpha$ / VEGF Axis. Front Pharmacol 2020;11:503524.

31. Li S, Zhou H, Hu C, et al. Total Flavonoids of Rhizoma Drynariae Promotes Differentiation of Osteoblasts and Growth of Bone Graft in Induced Membrane Partly by Activating Wnt/ $\beta$-Catenin Signaling Pathway. Front Pharmacol 2021;12:675470.

(English Language Editors: J. Goetz and J. Gray)

Cite this article as: Liu T, Huang J, Xu D, Li Y. Identifying a possible new target for diagnosis and treatment of postmenopausal osteoporosis through bioinformatics and clinical sample analysis. Ann Transl Med 2021;9(14):1154. doi 10.21037/atm-21-3098 